\title{
Analysis of Differentially Expressed Genes in Gastrocnemius Muscle between DGAT1 Transgenic Mice and Wild-Type Mice
}

\author{
Fei Ying, ${ }^{1}$ Hao Gu, ${ }^{1}$ Yuanzhu Xiong, ${ }^{1}$ and Bo Zuo ${ }^{1,2}$ \\ ${ }^{1}$ Key Laboratory of Swine Genetics and Breeding, Ministry of Agriculture and Key Lab of \\ Agricultural Animal Genetics and Breeding, Ministry of Education, College of Animal Science and Veterinary Medicine, \\ Huazhong Agricultural University, Wuhan 430070, China \\ ${ }^{2}$ The Cooperative Innovation Center for Sustainable Pig Production, Wuhan 430070, China \\ Correspondence should be addressed to Bo Zuo; zuobo@mail.hzau.edu.cn
}

Received 28 October 2016; Accepted 22 January 2017; Published 13 March 2017

Academic Editor: Young-Mi Lee

Copyright (C) 2017 Fei Ying et al. This is an open access article distributed under the Creative Commons Attribution License, which permits unrestricted use, distribution, and reproduction in any medium, provided the original work is properly cited.

\begin{abstract}
Adipose tissue was the major energy deposition site of the mammals and provided the energy for the body and released the external pressure to the internal organs. In animal production, fat deposition in muscle can affect the meat quality, especially the intramuscular fat (IMF) content. Diacylglycerol acyltransferase-1 (DGAT1) was the key enzyme to control the synthesis of the triacylglycerol in adipose tissue. In order to better understand the regulation mechanism of the DGAT1 in the intramuscular fat deposition, the global gene expression profiling was performed in gastrocnemius muscle between DGAT1 transgenic mice and wild-type mice by microarray. 281 differentially expressed transcripts were identified with at least 1.5-fold change and the $p$ value $<$ 0.05. 169 transcripts were upregulated and 112 transcripts were downregulated. Ten genes (SREBF1, DUSP1, PLAGL1, FKBP5, ZBTB16, PPP1R3C, CDC14A, GLUL, PDK4, and UCP3) were selected to validate the reliability of the chip's results by the real-time PCR. The finding of RT-PCR was consistent with the gene chip. Seventeen signal pathways were analyzed using KEGG pathway database and the pathways concentrated mainly on the G-protein coupled receptor protein signaling pathway, signal transduction, oxidation-reduction reaction, olfactory receptor activity, protein binding, and zinc ion binding. This study implied a function role of DGAT1 in the synthesis of TAG, insulin resistance, and IMF deposition.
\end{abstract}

\section{Introduction}

Adipose tissue was the major energy deposited site of the mammals. Also, it provided the energy for the body, kept the temperature stable, and released the external pressure $[1,2]$. Adipose tissue can be divided into subcutaneous fat, visceral fat, intermuscular fat, and intramuscular fat by the different deposition site. The intramuscular fat (IMF) was deposited in the muscle tissue [3]. It was the latest formation in adipose tissue. It affected the quality, tenderness, and color of the livestock's production [4-6]. Intramuscular fat was composed of structural fat, phospholipids, and triacylglycerol.

The triacylglycerol (TAG) was the major component of intramuscular fat and it was the important storage molecule of metabolic energy [7, 8]. TAG was one type of neutral lipid, which had a glycerol backbone and three long fatty acids. In animal, the TAG was always in the liver, small intestine, muscle, and adipose tissue. TAG was important for the cell membrane composition and lipoprotein transportation [9]. There were two pathways in the synthesis of TAG [10]. One relied on the acyl-CoA and the other not. The main pathway of TAG synthesis relied on the acyl-CoA [11, 12].

In adipose tissue, the acyl-CoA:diacylglycerol acyltransferase (DGAT) enzyme was the main catalyzer in the last and the only committed step of the major pathway of TAG synthesis $[13,14]$. The DGAT enzyme had two isoforms: DGAT1 and DGAT2. DGAT1 was a member of a large family of membrane-bound O-acyltransferases (MBOAT), whereas DGAT2 was a new family [15]. Both the DGAT1 and DGAT2 were the key enzyme in the TAG synthesis, but they had the distinguished function $[16,17]$. To determine the biological functions of DGAT1, Chen et al. $[18,19]$ created the DGAT1deficient mice. The mice lacking DGAT1 showed significant change in lipid metabolism in several tissues. The DGAT1deficient mice were resistant to obesity and had increased sensitivity to insulin and leptin. The effects of DGAT1 
deficiency on energy and glucose metabolism resulted in part from the altered secretion of adipocyte-derived factors [20, 21]. Otherwise, Buhman et al. [22] used the DGAT1deficient mice to analyze the triacylglycerol absorption and chylomicron synthesis. They find that DGAT1 is not essential for quantitative dietary triacylglycerol absorption, even in mice fed a high fat diet, or for the synthesis of chylomicrons. Smith et al. [23] demonstrated that DGAT1-deficient mice were viable and can still synthesize triglycerides. The finding indicated that multiple mechanisms exist for triglyceride synthesis. On the contrary, DGAT1 overexpressed in mice increased DGAT1 activity with threefold compared with the WT mice. The overexpression of DGAT1 caused the significant change in fat metabolism, including raised triglyceride synthesis, enhanced fatty acid oxidation, and preserved insulin sensitivity $[24,25]$.

Research over the past 20 years had predominantly focused on protein coding messenger RNA transcripts and their role in cellular processes, such as disease and development. These whole-transcript array designs provided a complete expression profile of mRNA that impact the mRNA expression profile. $\mathrm{Li}$ et al. [26] demonstrated that the overexpression of DGAT1 in the DGAT1 transgenic mice can increase the synthesis of TAG and IMF content, but the mechanism is not clear. In order to better understand and find the possible signal pathway of the DGAT1 regulating the intramuscular fat deposition, we use microarray technology to screen the differentially expressed genes in gastrocnemius muscle between DGAT1 transgenic mice and wild-type mice in this study.

\section{Materials and Methods}

2.1. Plasmid and Transgenic Mice. The construct (MCKDGAT1) contains, from the $5^{\prime}$-end to the $3^{\prime}$-end, a $7.0 \mathrm{~kb}$ porcine MCK promoter, obtained by homologous recombination from porcine bacterial artificial chromosome (GenBank accession number AC139878, Sus scrofa clone RP44251A2), and a $1.4 \mathrm{~kb}$ porcine DGAT1 cDNA without $5^{\prime} \mathrm{UTR}$ (untranslated region) and $3^{\prime} \mathrm{UTR}$, obtained by gene synthesis (GenBank accession number NM_214051.1, Invitrogen, Shanghai, China). TG mice were generated by a standard DNA microinjection [27] of C57BL/6 (Invitrogen, Shanghai, China). Founder TG mice were allowed to mate with wildtype (WT) mice and gave birth to the F1 generation of TG mice (identified by polymerase chain reaction- (PCR-) based genotyping) (see Figure S1 in the Supplementary Material available online at https://doi.org/10.1155/2017/5404682). Three-month F1 generation male mice gastrocnemius muscles were used in this study. All mice were kept at room temperature $\left(22^{\circ} \mathrm{C}\right)$ with a $12 \mathrm{~h}$ light/dark cycle. Mice were ad libitum fed a chow diet. All procedures were in accordance with institution guidelines and approved by the Institutional Animal Care and Use Committee of Hubei Province.

2.2. Reverse Transcriptase PCR and Real-Time PCR Analysis. The mRNA was extracted from gastrocnemius muscle samples using the RNeasy Lipid Tissue Mini kit (Qiagen, Hilden,
Germany) according to manufacturer's protocols. RNA quality was examined using gel electrophoresis (Figure S2). Total RNA were treated with RNase-free DNase I and subsequently used as a template for first-strand cDNA synthesis using a Revert Aid First Stand cDNA Synthesis Kit (Fermentas Inc., Glen Burnie, MD). Real-time PCR was performed with the SYBR qPCR Mix (Toyobo, Osaka, Japan) in a Bio-Rad CFX96 Real-Time PCR system. The primers sequences were listed in Table 1 . The extension time was $30 \mathrm{~s}$. Data were analyzed by the comparative critical threshold method [28], normalized by the amount of $\beta$-actin mRNA, and expressed relative to the corresponding value in WT mice.

2.3. Microarray Experiments. The mRNA was processed for hybridization to Affymetrix mouse gene 2.0 ST arrays (Affymetrix, Santa Clara, CA). These arrays provided wholetranscript coverage, with each of 26,515 genes represented on the array by approximately 27 probes spread across the full length of the gene. A transcript was called detectable if it had more than 7 detected probes. A total of six arrays were used (3 DGAT1 transgenic mice and 3 WT mice), and each array corresponded to labeled RNA from one individual gastrocnemius muscle. The data had submitted to the GEO and the accession number is GSE89192.

2.4. Data Analysis. The expression values for each gene were acquired using the GeneChip Operating System (GCOS 1.4, Affymetrix). The expression data from six mice were loaded into Gene-Spring GX 10.0 software (Agilent Technologies) for data normalization and filtering, which were differentially expressed between transgenic animals and control animals. Significance $(p<0.05)$ was calculated using an analysis of variance (ANOVA). Differentially expressed transcripts were identified by cutoff of fold change (FC) $\geq 1.5$ and $p$ value $<$ 0.05 using unpaired $t$-test.

2.5. Bioinformatics Analysis. The gene functions were determined primarily using the NCBI Entrez Gene database. Hierarchical cluster (Ver.2.11) was performed for differentially expressed genes [29]. In order to identify molecular interactions among the genes, Gene Ontology (GO) and pathwaybased analysis was carried out on differentially expressed genes to explore if there were significant enrichments of functional categories. Gene Ontology contains three different categories: biological process, cellular components, and molecular function, which were selected to investigate the molecular function of differentially expressed genes. Molecular function of differentially expressed genes was classified according to MAS (molecule annotation system) 3.0 platform (http://bioinfo.capitalbio.com/mas3/). Kyoto Encyclopedia of Genes and Genomes (KEGG) database were used for pathway analysis of differentially expressed genes.

2.6. Statistical Analyses. The data were analyzed by leastsquare analysis of variance procedures using SPSS16.0.0 with fixed effects of genotype and their interaction. We used unpaired 2-tailed Student's $t$-test to evaluate statistical significance. $p<0.05$ was considered as statistical significant. 
TABLE 1: Primer information of differentially expressed genes used for real-time PCR.

\begin{tabular}{|c|c|c|c|}
\hline Gene name & Primer sequences $\left(5^{\prime}-3^{\prime}\right)$ & $\operatorname{Tm}\left({ }^{\circ} \mathrm{C}\right)$ & Product size \\
\hline SREBF1 & $\begin{array}{l}\text { CAGAACTGGGACCTTGGGAC } \\
\text { CCCAGCTCCTCTGTCTTTGG }\end{array}$ & 60 & 162 \\
\hline UCP3 & $\begin{array}{l}\text { CTGCACCGCCAGATGAGTTT } \\
\text { ATCATGGCTTGAAATCGGACC }\end{array}$ & 60 & 191 \\
\hline DUSP1 & $\begin{array}{l}\text { AGTGCCTATCACGCTTCTCG } \\
\text { GGAGCTGATGTCTGCCTTGT }\end{array}$ & 58 & 153 \\
\hline FKBP5 & $\begin{array}{l}\text { CTGCACCGCCAGATGAGTTT } \\
\text { AAAGAAAAGCTGACGCAGGC }\end{array}$ & 60 & 140 \\
\hline ZBTB16 & $\begin{array}{l}\text { CTGGGACTTTGTGCGATGTG } \\
\text { CGGTGGAAGAGGATCTCAAACA }\end{array}$ & 60 & 106 \\
\hline PDK4 & $\begin{array}{l}\text { AGGGAGGTCGAGCTGTTCTC } \\
\text { GGAGTGTTCACTAAGCGGTCA }\end{array}$ & 60 & 185 \\
\hline GLUL & $\begin{array}{l}\text { CAACGACTTTTCTGCCGGTG } \\
\text { TATTGGAAGGGTTCGTCGCC }\end{array}$ & 59 & 186 \\
\hline PLAGL1 & $\begin{array}{l}\text { CACCTCACTCGTCACACCAA } \\
\text { TGAAGGCGCAATGAGTTGGA }\end{array}$ & 60 & 102 \\
\hline CDC14A & $\begin{array}{l}\text { GATAACATCGTGCGGAGATTCC } \\
\text { CATAACAGGCTATCAATGTCCCG }\end{array}$ & 60 & 109 \\
\hline PPP1R3C & $\begin{array}{l}\text { TGCAATGGAAACCTGACGGA } \\
\text { AAGTTCTCCACTCTCCCCCA }\end{array}$ & 60 & 163 \\
\hline$\beta$-Actin & $\begin{array}{l}\text { AGGCCCAGAGCAAGAGAGGTA } \\
\text { GGGGTGTTGAAGGTCTCAAACA }\end{array}$ & 60 & 220 \\
\hline
\end{tabular}

\section{Results and Discussion}

3.1. Assessment of the Microarray Quality. We used the PARTEK to analyze the value of the chip signal after homogenization. The picture showed that the signal value distribution of each chip was consistent (Figure S3). The linear combination of three variables with the highest proportion in the total probe cluster demonstrated that there were significant differences between the transgenic mice $(n=3)$ and the wild-type mice $(n=3)$ (Figure S4). According to the hierarchical clustering (Figure S5), we found that three transgenic mice were clustered in one group and three WT mice were in another group.

3.2. The Screening of Differentially Expressed Genes. In the gene microarray chip results, 281 differentially expressed transcripts were identified with at least 1.5 -fold change and the $p$ value $<0.05$. In the 281 transcripts, 169 transcripts were upregulated and 112 were downregulated. By checking the RefSeq in the database, we find that 71 transcripts have the annotation (43 were upregulated and 28 were downregulated) (Table S1).

3.3. Validation of Gene Expression Data by Real-Time PCR. To validate the results of the gene chips, RT-PCR was carried out for three upregulated genes (SREBF1, DUSP1, and PLAGL1) and seven downregulated genes (FKBP5, ZBTB16, PPP1R3C, CDC14A, GLUL1, PDK4, and UCP3). As shown in Figure 1, the relative fold differences in the gene expression as determined by the RT-PCR were similar to the results of the microarray analysis. Meanwhile, the $p$ value is less than 0.05 and $r^{2}$ is more than 0.5 .

3.4. The Signal Pathway Analysis. To categorize the differentially expressed genes, we used the Kyoto Encyclopedia of Genes and Genomes (KEGG) analysis. It showed that the genes were in lots of signal pathways (Table 2), such as the insulin signaling pathway, the PPAR signaling pathway, and the biosynthesis of unsaturated fatty acids pathway. Otherwise, we find that several important genes simultaneously participated in some biological process and signal pathways by analyzing the network of the signal pathway and genes (Figure 2). As shown in Figure 2, the Glul regulated the glutamate metabolism, the nitrogen metabolism, and peptidoglycan biosynthesis.

3.5. The Gene Ontology (G0) Classification. The GO analysis assigned different genes to the different kind of categories. By importing the data into the MAS 3.0 database, all the genes were analyzed together without distinguishing upregulated and downregulated genes. In the results (Figure 3), 78 genes $(47.56 \%)$ were classified into biological process, 63 genes (38.41\%) were in molecular function, and 23 genes $(14.02 \%)$ were in cellular component.

3.6. Interaction Analysis of Differentially Expressed Genes Encoding Proteins. We used four databases (MINT, HPRD, InAct, and DIP) supported by the MAS 3.0 to analyze the interaction between the proteins which were the differentially 
TABLE 2: The pathway information of some differentially expressed genes.

\begin{tabular}{|c|c|c|}
\hline Pathway & Gene symbol & Gene title \\
\hline Olfactory transduction & $\begin{array}{c}\text { Olfr1445 } \\
\text { Olfr350 } \\
\text { Olfr746 } \\
\text { Olfr311 } \\
\text { Olfr1028 } \\
\text { Olfr1420 } \\
\text { Olfr1006 }\end{array}$ & $\begin{array}{c}\text { Olfactory receptor } 1445 \\
\text { Olfactory receptor } 350 \\
\text { Olfactory receptor } 746 \\
\text { Olfactory receptor } 311 \\
\text { Olfactory receptor } 1028 \\
\text { Olfactory receptor } 1420 \\
\text { Olfactory receptor } 1006\end{array}$ \\
\hline \multirow[t]{2}{*}{ Insulin signaling pathway } & Srebf1 & $\begin{array}{c}\text { Sterol regulatory element } \\
\text { binding transcription factor } 1\end{array}$ \\
\hline & Ppplr3c & $\begin{array}{l}\text { Protein phosphatase 1, regulatory } \\
\text { (inhibitor) subunit }\end{array}$ \\
\hline Peptidoglycan biosynthesis & Glul & Glutamate-ammonia ligase \\
\hline Terpenoid biosynthesis & Sqle & Squalene epoxidase \\
\hline Nitrogen metabolism & Glul & Glutamate-ammonia ligase \\
\hline Biosynthesis of steroids & Sqle & Squalene epoxidase \\
\hline $\begin{array}{l}\text { Biosynthesis of unsaturated fatty } \\
\text { acids }\end{array}$ & $\operatorname{Scd} 2$ & $\begin{array}{c}\text { Stearoyl-coenzyme A desaturase } \\
2\end{array}$ \\
\hline Glutamate metabolism & Glul & Glutamate-ammonia ligase \\
\hline $\begin{array}{l}\text { Porphyrin and chlorophyll } \\
\text { metabolism }\end{array}$ & Hccs & Holocytochrome-c synthetase \\
\hline Acute myeloid leukemia & Zbtb16 & $\begin{array}{l}\text { Zinc finger and BTB domain } \\
\text { containing } 16\end{array}$ \\
\hline PPAR signaling pathway & $\operatorname{Scd} 2$ & $\begin{array}{l}\text { Stearoyl-coenzyme A desaturase } \\
2\end{array}$ \\
\hline Ribosome & Rps3a & Ribosomal protein S3A \\
\hline Cell cycle & Cdc14a & CDC14 cell division cycle 14A \\
\hline Ubiquitin mediated proteolysis & Ubely1 & $\begin{array}{l}\text { Ubiquitin-activating enzyme E1, } \\
\text { Chr Y } 1\end{array}$ \\
\hline Parkinson's disease & Ubelyl & $\begin{array}{l}\text { Ubiquitin-activating enzyme E1, } \\
\text { Chr Y } 1 \\
\end{array}$ \\
\hline $\begin{array}{l}\text { Cytokine-cytokine receptor } \\
\text { interaction }\end{array}$ & Ccr3 & $\begin{array}{c}\text { Chemokine (C-C motif) receptor } \\
3\end{array}$ \\
\hline MAPK signaling pathway & Dusp1 & Dual specificity phosphatase 1 \\
\hline
\end{tabular}

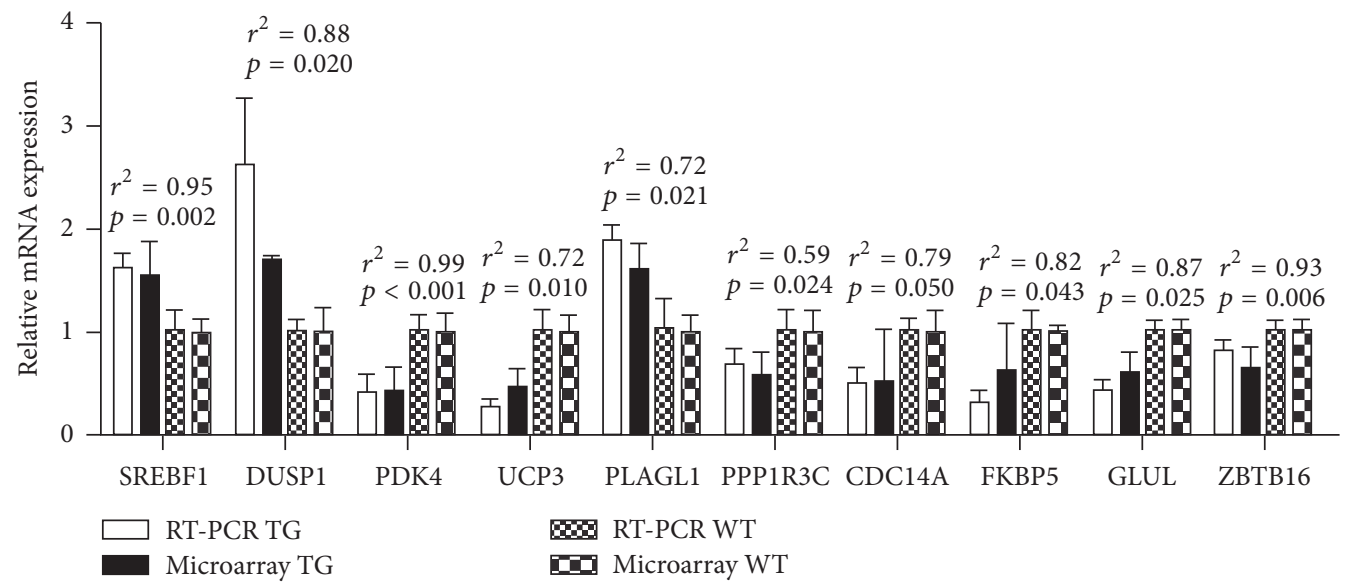

FIGURE 1: The verification result of microarray by QRT-PCR. The $x$-axis represents the genes; the $y$-axis represents the relative mRNA expression. 


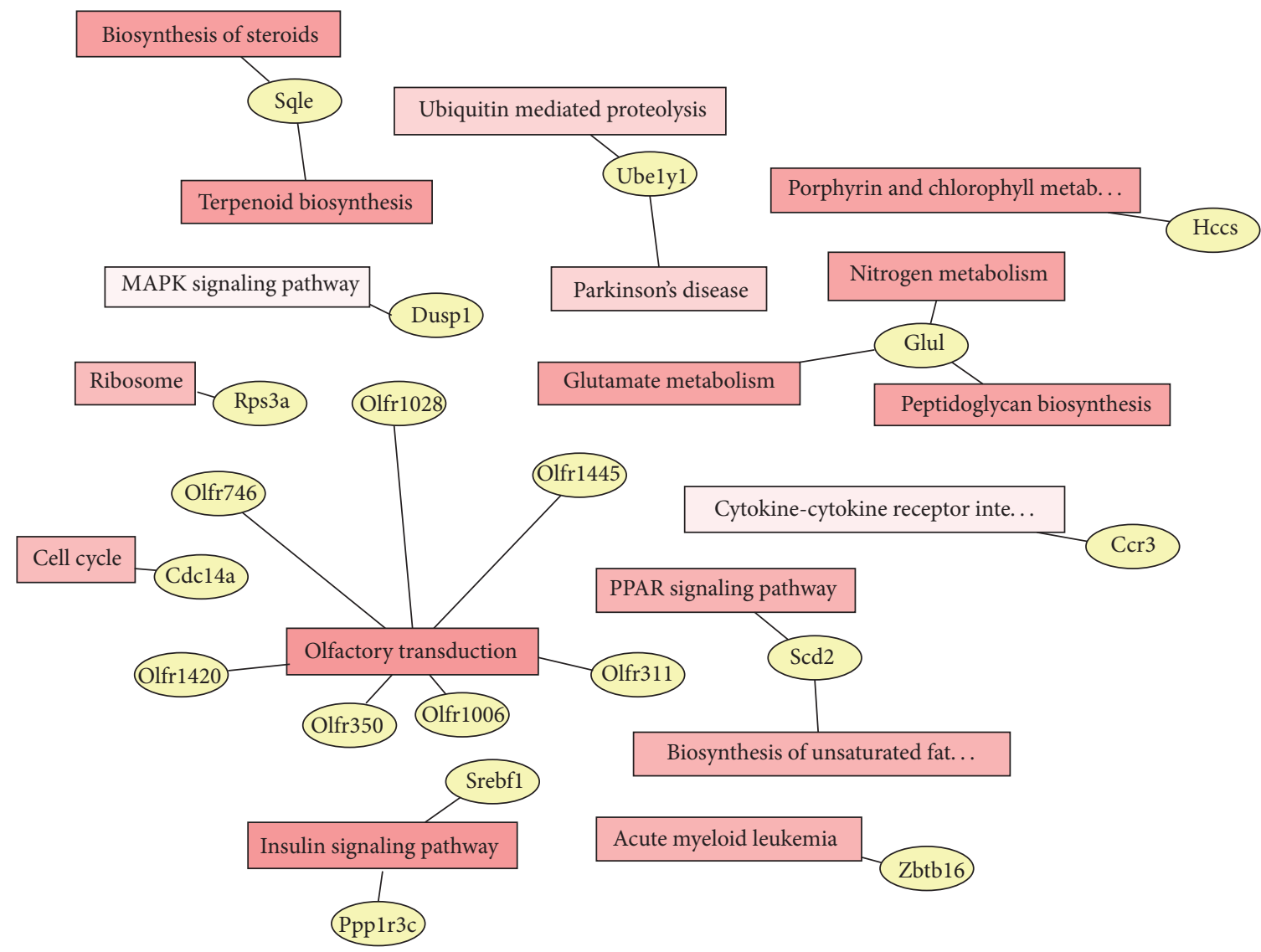

Figure 2: Pathway network of differentially expressed genes. The yellow elliptical box means the differentially expressed genes and the quadrate box means the signal pathway.

expressed genes translated (Figure 4). The Fkbp5 protein interacted with 2 subtypes of the $\mathrm{Nr} 3 \mathrm{cl}$ protein.

3.7. Detection of the Global Gene Expression in the Gastrocnemius of the DGAT1 Transgenic Mice and WT Mice by Microarray. In our study, the gene expression differences were processed by the Affymetrix mouse gene 2.0 st. Microarray data revealed that many genes were more than 1.5 -fold change and $p<0.05$ ( 43 were upregulated and 28 were downregulated). Ten genes (SREBF1, DUSP1, PLAGL1, FKBP5, ZBTB16, PPP1R3C, CDC14A, GLUL, PDK4, and UCP3) were selected for validation by RT-PCR. All of them show the significant differential expression level. Furthermore, the trends were same between the results of two methods, showing the reliability of the microarray analysis. It is reasonable to expect that these genes were associated with the DGAT function.

The GO analysis demonstrated that three major kinds of categories are connected with the differentially expressed genes, including G-protein coupled receptor protein signal pathway, receptor activity, and integral membrane. Meanwhile, the KEGG pathway showed that some of genes were involved in the insulin signal pathway (SREBF1, PDK4, and PPP1R3C), the MAPK signaling pathway (DUSP1), and the biosynthesis of unsaturated fatty acids pathway (SCD2, UCP3).

3.8. The Differentially Expressed Genes Associated with the Glucose Metabolism. Pyruvate dehydrogenase complex (PDC) was a key enzyme in the process of tricarboxylic acid cycle, which can promote the decarboxylation of the acetyl CoA [30]. However, it was inhibited by pyruvate dehydrogenase kinase (PDK). PDK4 was a subtype of the PDK family. PDK4 can regulate the synthesis of glycine and the production of glycolysis [31]. Wang et al. discovered that knocking out the PDK4 could decrease the level of blood sugar and improve the tolerability of the glucose. In our study, the PDK4 in the DGAT1-overexpression mice was decreased by 2.4 -fold. This indicated that the overexpression of DGAT1 could relieve the inhibition of PDC by downregulating the PDK4 and then promote the process of TAG synthesis. At last, the glucose utilization was increased and insulin resistance was avoided.

PP1 family was a phosphoric acid protease family. It can regulate glycogen synthetase (GS) and glycogen phosphorylase (GP) activity and control the synthesis and decomposition of the glycogen [32]. As a member of the PP1 family, 


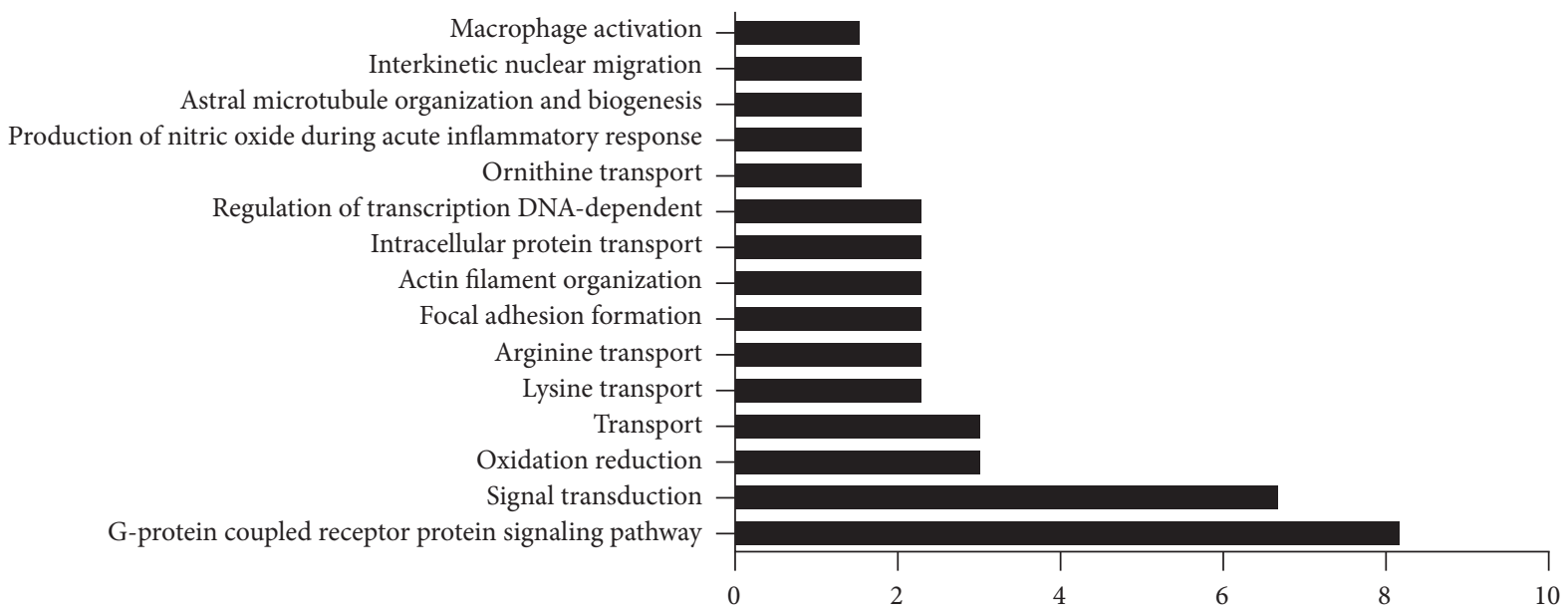

(a) Biological process-all differential genes in TG mice

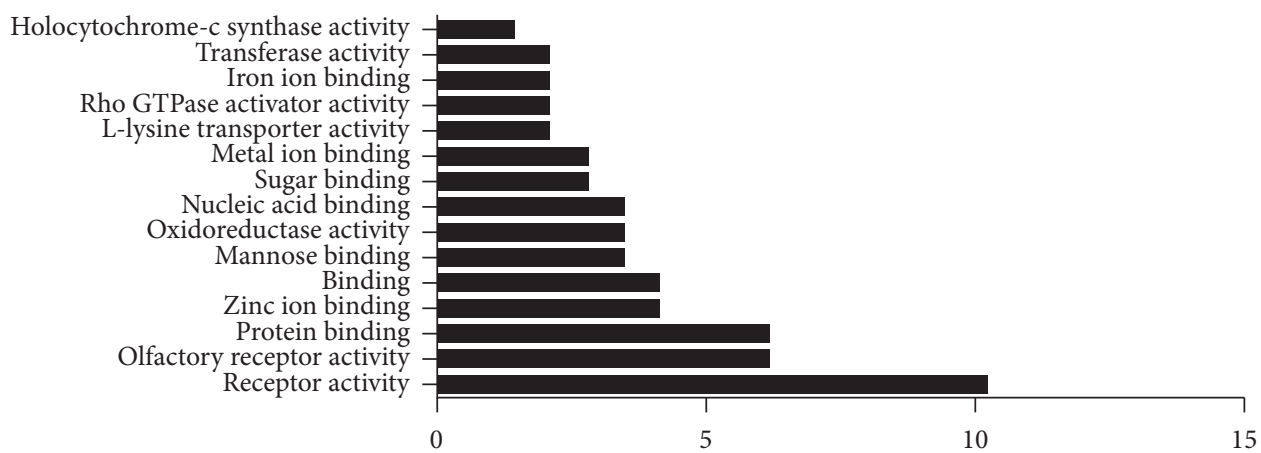

(b) Molecular function-all differential genes in TG mice

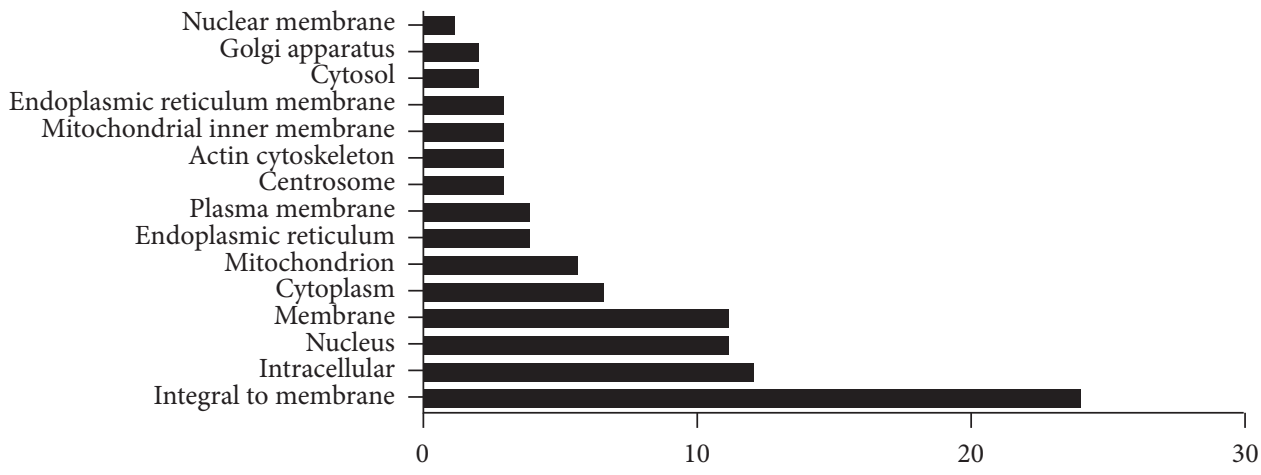

(c) Cellular component-all differential genes in TG mice

FIGURE 3: Gene ontology analysis of all differentially expressed genes.

PPP1R3C was expressed mainly in the muscle and liver. PPP1R3C participated in glucose metabolism and glycogen synthesis. The high glucose stimulated its expression [33]. In our experiment, the PPP1R3C was 1.7-fold downregulated. So we inferred that DGAT1 regulated the insulin sensitivity and the metabolism of glucose by the PPP1R3C.

3.9. The Differentially Expressed Genes Associated with the Lipogenesis. Sterol regulatory element binding protein (SREBF1) is a membrane binding protein. It can be in the role of proteases shuttle in the nucleus and induce the lipogenesis gene expression [34]. SREBF1 can regulate the synthesis of the fatty acid and sterols. Also, it was the key factor of the TAG metabolism and can regulate the lipid metabolism by the insulin signaling pathway [35]. The upexpression of SREBF1 in goat mammary epithelial cells can increase DGAT1 and LPIN1, promoting the synthesis of fatty acids and the deposition of TAG [36]. In the microarray data, the mRNA level of the SREBF1 increased more than 1.5-fold in transgenic mice, revealing that the overexpression of DGAT1 can induce the SREBF1. Furthermore, the DGAT1 increased the content of TAG, affected the sensibility of insulin, and adjusted the body's energy metabolism by the SREBF1. 


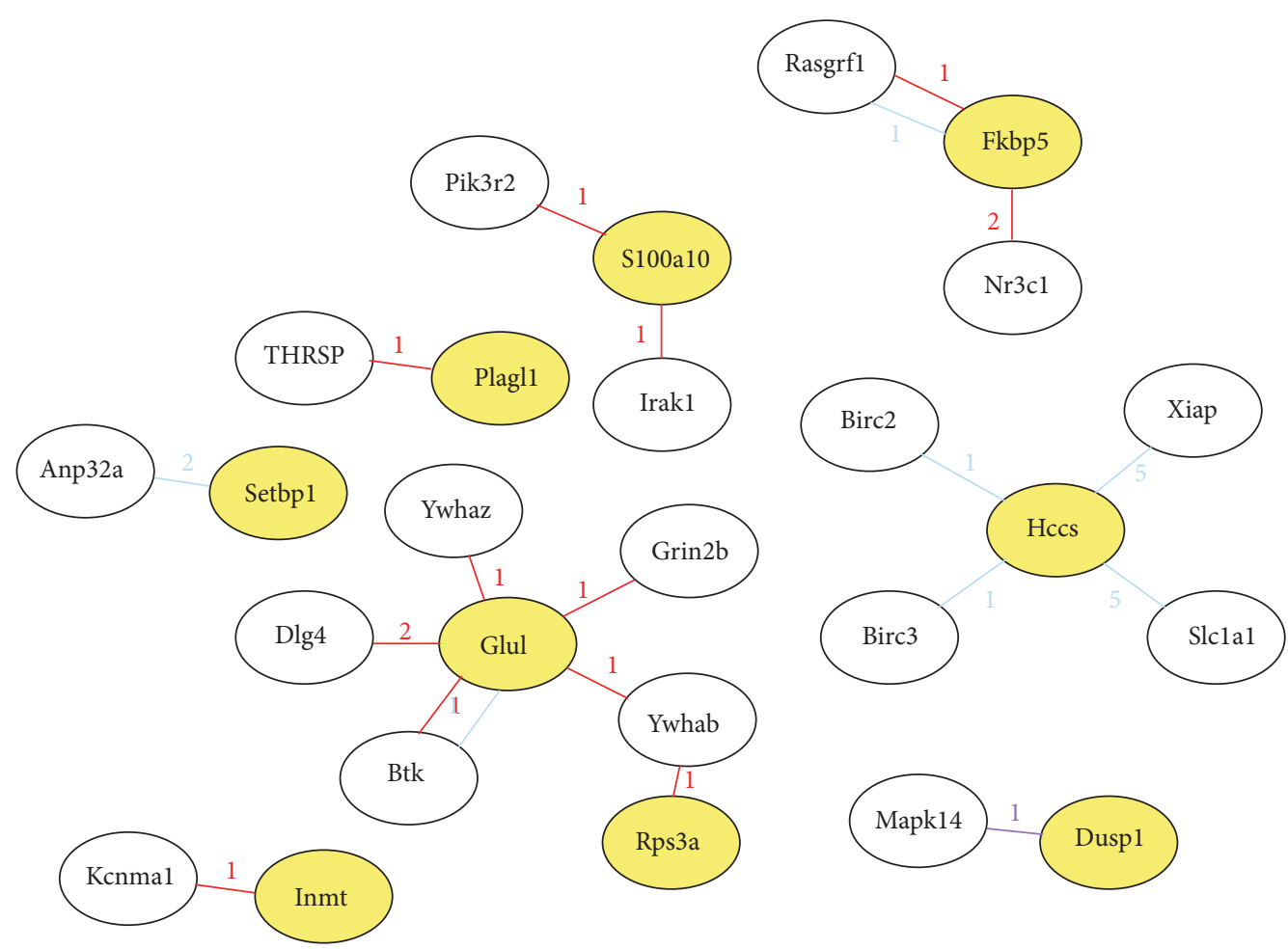

FIGURE 4: Network of protein interaction. Red line represents InAct database, blue line represents MINT database, and purple line represents DIP database. The number on the line means the subtype of the protein.

Further studies will be needed to test the protein level of the SREBF1 and find the mechanism between the DGAT1 and SREBF1.

Stearoyl-coenzyme A desaturase (SCD) was a key enzyme that catalyzed the conversion of saturated fatty acids to unsaturated fatty acids, which can regulate the percentage of saturated fatty acids and unsaturated fatty acids in cells. It had 4 subtypes (SCD1, SCD2, SCD3, and SCD4) in the mouse. SCD2 affected the body's energy metabolism by regulating the synthesis of fatty acid and glycolysis through the PPAR signaling pathway. PPAR, as a key factor of lipid synthesis, increased the expression of adiponectin and TAG related genes to regulate glucose utilization and insulin sensitivity [37]. In our study, the expression of SCD 2 was upregulated by 1.7-fold in transgenic mice. SCD1 also increased more than 2fold, but it was not significant. This change demonstrated that DGAT1 facilitate the formation of fat and energy metabolism by regulating the genes in the PPAR signaling pathway.

3.10. The Differentially Expressed Genes Associated with the Lipid Oxidation. Dual specificity phosphatase 1 (DUSP1) was the member of the MKP phosphatase family. It regulated the MAPK signaling pathway by the dephosphorylation. DUSP1deficient mice increased energy output to avoid the obesity caused by the high fat diet $[38,39]$. DUSP1 can directly inhibit PGC-1 alpha by the p38-MAPK pathway. Furthermore, DUSP1 changed the muscle fiber type by PGC-1 alpha, thus affecting the composition of muscle fiber [40]. DUSP1 can also affect the metabolism of fatty acids. Knocking out DUSP1 can enhance the oxidation of fatty acids and control the body's energy output [41]. In our data, the expression of DUSP1 increased 1.7-fold in transgenic mice, indicating the overexpression of DGAT1 can upregulate DUSP1. And it can promote the dephosphorylation of the p38-MAPK and inhibit the synthesis of mitochondria. In particular, the upregulation of DUSP1 can enhance the expansion of type I muscle fiber by PGC- $1 \alpha$. As we know, the type I muscle fiber had a higher content of TAG and glucose transporter 4 (GLUT4). So, the overexpression of DGAT1 regulated the synthesis of TAG and energy metabolism by the MAPK signaling pathway and PGC- $1 \alpha$. Therefore, we think that DGAT1 controlled the deposition of fat by the DUSP1.

Uncoupling protein 3 (UCP3) belonged to the mitochondrial carrier protein family and was mainly expressed in skeletal muscle and brown adipose tissue. UCP3 was mainly involved in the uncoupling effect of mitochondrial respiratory chain and played an important role in the maintenance of mitochondrial activity, the adaptability of the body thermogenesis, and the fatty acid oxidation [42, 43]. The overexpression of UCP3 increased the feed intake and reduced blood glucose and insulin levels in mice [44]. Accumulating the more fatty acids in the liver cells induced UCP3 expression. UCP3 activated fatty acid metabolism genes, changed the permeability of the mitochondrial inner membrane, and reduced the damage of liver cell. In the study, UCP3 decreased more than 2.0-fold. This result showed that the energy output of DGAT1 transgenic mice was relatively low, and the fat deposition was faster. This was consistent with 
the bigger body size of DGAT1 transgenic mice as discovered previously, comparing with the WT mice. So the DGAT1 can increase the level of insulin in the blood and the body weight and fat by the UCP3.

Zinc finger and BTB domain containing (ZBTB) 16 was a transcriptional inhibitor, belonging to the proteins' superfamily. The expression levels of ZBTB16 in brown adipose tissue and skeletal muscle were 15.3 and 2.1 times higher in cold-stimulated mice, respectively. The overexpression of ZBTB16 increased the number of mitochondria and the glucose consumption and reduced the content of the TAG in the cell. In our study, the mRNA level of ZBTB16 decreased 1.5 -fold. It revealed that DGAT1 can reduce the oxidation of fatty acids and the energy output of the body by the ZBTB16, which were better for the deposition of intramuscular fat.

Taken together, glucose and lipid metabolism was regulated and the reaction of the TAG synthesis was catalyzed by the overexpression of DGAT1. Afterwards, the TAG synthesis was sufficient to improve muscle insulin sensitivity and increase the content of IMF. As a result, the upregulation of DGAT1 led to insulin sensitivity and IMF improvement.

\section{Conclusion}

In our study, we used the microarray to detect the differential gene expression in the three-month DGAT1 transgenic mice and WT mice. Furthermore, we found 7 differentially expressed genes involved in glucose metabolism, lipogenesis, and lipid oxidation, which were found to be connected with IMF content. As the previous evidences show, SREBF1, UCP3, and SCD2 participated in the DGAT1 regulation, which was consistent with our results. Moreover, there were no reports about the regulation of DUSP1, PPP1R3C, PDK4, and ZBTB16 by DGAT1 until now. So these genes identified in our study merit further investigation to understand the signal pathway of the DGAT1 regulating the synthesis of TAG and the formation of IMF. Thus, we inferred that DGAT1 regulates the synthesis of TAG and the formation of IMF by possibly affecting the insulin signaling pathway, the MAPK signaling pathway, the biosynthesis of unsaturated fatty acids, and so on. This research may open a possible means to inhibit insulin resistance and treat type 2 diabetes.

\section{Competing Interests}

The authors declare that they have no competing interests.

\section{Acknowledgments}

The study was financially supported by grants from the National Key Technology Support Program of China (Grant no. 2014BAD20B01), the National Project for Breeding of Transgenic Pig (2016ZX08006-002), the Agricultural Innovation Fund of Hubei Province (Grant no. 2007-620), and the Fundamental Research Funds for the Central Universities (Grant nos. 2014PY038 and 2662016PY012).

\section{References}

[1] E. E. Kershaw and J. S. Flier, "Adipose tissue as an endocrine organ," Journal of Clinical Endocrinology and Metabolism, vol. 89, no. 6, pp. 2548-2556, 2004.

[2] C. M. O. do Nascimento, E. B. Ribeiro, and L. M. Oyama, "Metabolism and secretory function of white adipose tissue: effect of dietary fat," Anais da Academia Brasileira de Ciencias, vol. 81, no. 3, pp. 453-466, 2009.

[3] R. J. Richmond and R. T. Berg, "Tissue development in swine as influenced by live weight, breed, sex and ration," Canadian Journal of Animal Science, vol. 51, no. 1, pp. 31-39, 1971.

[4] H.-X. Cui, R.-R. Liu, G.-P. Zhao, M.-Q. Zheng, J.-L. Chen, and J. Wen, "Identification of differentially expressed genes and pathways for intramuscular fat deposition in pectoralis major tissues of fast-and slow-growing chickens," BMC Genomics, vol. 13, no. 1, article 213, 2012.

[5] M. Monziols, M. Bonneau, A. Davenel, and M. Kouba, “Tissue distribution in pig carcasses exhibiting large differences in their degree of leanness, with special emphasis on intermuscular fat," Livestock Production Science, vol. 97, no. 2-3, pp. 267-274, 2005.

[6] K. Suzuki, M. Ishida, H. Kadowaki, T. Shibata, H. Uchida, and A. Nishida, "Genetic correlations among fatty acid compositions in different sites of fat tissues, meat production, and meat quality traits in Duroc pigs," Journal of Animal Science, vol. 84, no. 8, pp. 2026-2034, 2006.

[7] B. Essén-Gustavsson and S. Fjelkner-Modig, "Skeletal muscle characteristics in different breeds of pigs in relation to sensory properties of meat," Meat Science, vol. 13, no. 1, pp. 33-47, 1985.

[8] A. Listrat, B. Lebret, I. Louveau et al., "How muscle structure and composition influence meat and flesh quality," The Scientific World Journal, vol. 2016, Article ID 3182746, 14 pages, 2016.

[9] R. A. Coleman and D. G. Mashek, "Mammalian triacylglycerol metabolism: synthesis, lipolysis, and signaling," Chemical Reviews, vol. 111, no. 10, pp. 6359-6386, 2011.

[10] S. Cases, S. J. Smith, Y.-W. Zheng et al., "Identification of a gene encoding an acyl CoA:diacylglycerol acyltransferase, a key enzyme in triacylglycerol synthesis," Proceedings of the National Academy of Sciences of the United States of America, vol. 95, no. 22, pp. 13018-13023, 1998.

[11] S. J. Stone, M. C. Levin, and R. V. Farese Jr., "Membrane topology and identification of key functional amino acid residues of murine Acyl-CoA:diacylglycerol acyltransferase-2," Journal of Biological Chemistry, vol. 281, no. 52, pp. 40273-40282, 2006.

[12] C.-L. E. Yen, D. W. Nelson, and M.-I. Yen, "Intestinal triacylglycerol synthesis in fat absorption and systemic energy metabolism," Journal of Lipid Research, vol. 56, no. 3, pp. 489501, 2015.

[13] M. Y. S. Shih, M. A. Kane, P. Zhou et al., "Retinol esterification by DGAT1 is essential for retinoid homeostasis in murine skin," Journal of Biological Chemistry, vol. 284, no. 7, pp. 4292-4299, 2009.

[14] H. Tomoda and S. Ōmura, "Potential therapeutics for obesity and atherosclerosis: inhibitors of neutral lipid metabolism from microorganisms," Pharmacology \& Therapeutics, vol. 115, no. 3, pp. 375-389, 2007.

[15] Y.-H. Yu and H. N. Ginsberg, "The role of acyl-CoA: diacylglycerol acyltransferase (DGAT) in energy metabolism," Annals of Medicine, vol. 36, no. 4, pp. 252-261, 2004.

[16] D. E. Kelley, B. H. Goodpaster, and L. Storlien, "Muscle triglyceride and insulin resistance," Annual Review of Nutrition, vol. 22, pp. 325-346, 2002. 
[17] R. L. Meegalla, J. T. Billheimer, and D. Cheng, "Concerted elevation of acyl-coenzyme A: diacylglycerol acyltransferase (DGAT) activity through independent stimulation of mRNA expression of DGAT1 and DGAT2 by carbohydrate and insulin," Biochemical and Biophysical Research Communications, vol. 298, no. 3, pp. 317-323, 2002.

[18] H. C. Chen, S. J. Smith, Z. Ladha et al., "Increased insulin and leptin sensitivity in mice lacking acyl CoA:diacylglycerol acyltransferase 1," Journal of Clinical Investigation, vol. 109, no. 8, pp. 1049-1055, 2002.

[19] H. C. Chen, S. J. Stone, P. Zhou, K. K. Buhman, and R. V. Farese Jr., "Dissociation of obesity and impaired glucose disposal in mice overexpressing acyl coenzyme A: diacylglycerol acyltransferase 1 in white adipose tissue," Diabetes, vol. 51, no. 11, pp. 31893195, 2002.

[20] C. L. Kelpe, L. M. Johnson, and V. Poitout, "Increasing triglyceride synthesis inhibits glucose-induced insulin secretion in isolated rat islets of Langerhans: a study using adenoviral expression of diacylglycerol acyltransferase," Endocrinology, vol. 143, no. 9, pp. 3326-3332, 2002.

[21] S. K. Koliwad, R. S. Streeper, M. Monetti et al., "DGAT1dependent triacylglycerol storage by macrophages protects mice from diet-induced insulin resistance and inflammation," Journal of Clinical Investigation, vol. 120, no. 3, pp. 756-767, 2010.

[22] K. K. Buhman, S. J. Smith, S. J. Stone et al., "DGAT1 is not essential for intestinal triacylglycerol absorption or chylomicron synthesis," Journal of Biological Chemistry, vol. 277, no. 28, pp. 25474-25479, 2002.

[23] S. J. Smith, S. Cases, D. R. Jensen et al., "Obesity resistance and multiple mechanisms of triglyceride synthesis in mice lacking Dgat," Nature Genetics, vol. 25, no. 1, pp. 87-90, 2000.

[24] L. Liu, X. Shi, S. C. Cheol et al., "Paradoxical coupling of triglyceride synthesis and fatty acid oxidation in skeletal muscle overexpressing DGAT1," Diabetes, vol. 58, no. 11, pp. 2516-2524, 2009.

[25] S. Timmers, J. de Vogel-van den Bosch, M. K. C. Hesselink et al., "Paradoxical increase in TAG and DAG content parallel the insulin sensitizing effect of unilateral DGAT1 overexpression in rat skeletal muscle," PLoS ONE, vol. 6, no. 1, Article ID e14503, 2011.

[26] T. Li, D. Xu, B. Zuo et al., "Ectopic overexpression of porcine DGAT1 increases intramuscular fat content in mouse skeletal muscle," Transgenic Research, vol. 22, no. 1, pp. 187-194, 2013.

[27] W. N. Drohan, D.-W. Zhang, R. Paleyanda et al., "Inefficient processing of human protein $\mathrm{C}$ in the mouse mammary gland," Transgenic Research, vol. 3, no. 6, pp. 355-364, 1994.

[28] K. J. Livak and T. D. Schmittgen, "Analysis of relative gene expression data using real-time quantitative PCR and the 2$\Delta \Delta$ CT method," Methods, vol. 25, no. 4, pp. 402-408, 2001.

[29] M. B. Eisen, P. T. Spellman, P. O. Brown, and D. Botstein, "Cluster analysis and display of genome-wide expression patterns," Proceedings of the National Academy of Sciences of the United States of America, vol. 95, no. 25, pp. 14863-14868, 1998.

[30] E. Saunier, C. Benelli, and S. Bortoli, "The pyruvate dehydrogenase complex in cancer: an old metabolic gatekeeper regulated by new pathways and pharmacological agents," International Journal of Cancer, vol. 138, no. 4, pp. 809-817, 2016.

[31] Y. Xie, H. Wang, X. Cheng et al., "Farnesoid X receptor activation promotes cell proliferation via PDK4-controlled metabolic reprogramming," Scientific Reports, vol. 6, Article ID 18751, 2016.
[32] J. Jensen and Y.-C. Lai, "Regulation of muscle glycogen synthase phosphorylation and kinetic properties by insulin, exercise, adrenaline and role in insulin resistance," Archives of Physiology and Biochemistry, vol. 115, no. 1, pp. 13-21, 2009.

[33] S. K. Lee, J. W. Moon, Y. W. Lee et al., "The effect of high glucose levels on the hypermethylation of protein phosphatase 1 regulatory subunit 3C (PPP1R3C) gene in colorectal cancer," Journal of Genetics, vol. 94, no. 1, pp. 75-85, 2015.

[34] J. Han, E. Li, L. Chen et al., "The CREB coactivator CRTC2 controls hepatic lipid metabolism by regulating SREBP1," Nature, vol. 524, no. 7564, pp. 243-246, 2015.

[35] X. Zhao, D. Feng, Q. Wang et al., "Regulation of lipogenesis by cyclin-dependent kinase 8 -mediated control of SREBP-1," Journal of Clinical Investigation, vol. 122, no. 7, pp. 2417-2427, 2012.

[36] H. F. Xu, J. Luo, W. S. Zhao et al., "Overexpression of SREBP1 (sterol regulatory element binding protein 1) promotes de novo fatty acid synthesis and triacylglycerol accumulation in goat mammary epithelial cells," Journal of Dairy Science, vol. 99, no. 1, pp. 783-795, 2016.

[37] J. L. Christianson, S. Nicoloro, J. Straubhaar, and M. P. Czech, "Stearoyl-CoA desaturase 2 is required for peroxisome proliferator-activated receptor $\gamma$ expression and adipogenesis in cultured 3T3-L1 cells," The Journal of Biological Chemistry, vol. 283, no. 5, pp. 2906-2916, 2008.

[38] J. J. Wu, R. J. Roth, E. J. Anderson et al., "Mice lacking MAP kinase phosphatase-1 have enhanced MAP kinase activity and resistance to diet-induced obesity," Cell Metabolism, vol. 4, no. 1, pp. 61-73, 2006.

[39] A. Khadir, A. Tiss, J. Abubaker et al., "Map kinase phosphatase duspl is overexpressed in obese humans and modulated by physical exercise," American Journal of PhysiologyEndocrinology and Metabolism, vol. 308, no. 1, pp. E71-E83, 2015.

[40] R. J. Roth, A. M. Le, L. Zhang et al., "MAPK phosphatase-1 facilitates the loss of oxidative myofibers associated with obesity in mice," The Journal of Clinical Investigation, vol. 119, no. 12, pp. 3817-3829, 2009.

[41] R. J. R. Flach, H. Qin, L. Zhang, and A. M. Bennett, "Loss of mitogen-activated protein kinase phosphatase-1 protects from hepatic steatosis by repression of cell death-inducing DNA Fragmentation Factor A (DFFA)-like effector C (CIDEC)/fatspecific protein 27," Journal of Biological Chemistry, vol. 286, no. 25, pp. 22195-22202, 2011.

[42] E. L. Seifert, V. Bézaire, C. Estey, and M.-E. Harper, "Essential role for uncoupling protein-3 in mitochondrial adaptation to fasting but not in fatty acid oxidation or fatty acid anion export," The Journal of Biological Chemistry, vol. 283, no. 37, pp. 2512425131, 2008.

[43] J. Himms-Hagen and M.-E. Harper, "Physiological role of UCP3 may be export of fatty acids from mitochondria when fatty acid oxidation predominates: an hypothesis," Experimental Biology \& Medicine, vol. 226, no. 2, pp. 78-84, 2001.

[44] M. D. Brand and T. C. Esteves, "Physiological functions of the mitochondrial uncoupling proteins UCP2 and UCP3," Cell Metabolism, vol. 2, no. 2, pp. 85-93, 2005. 

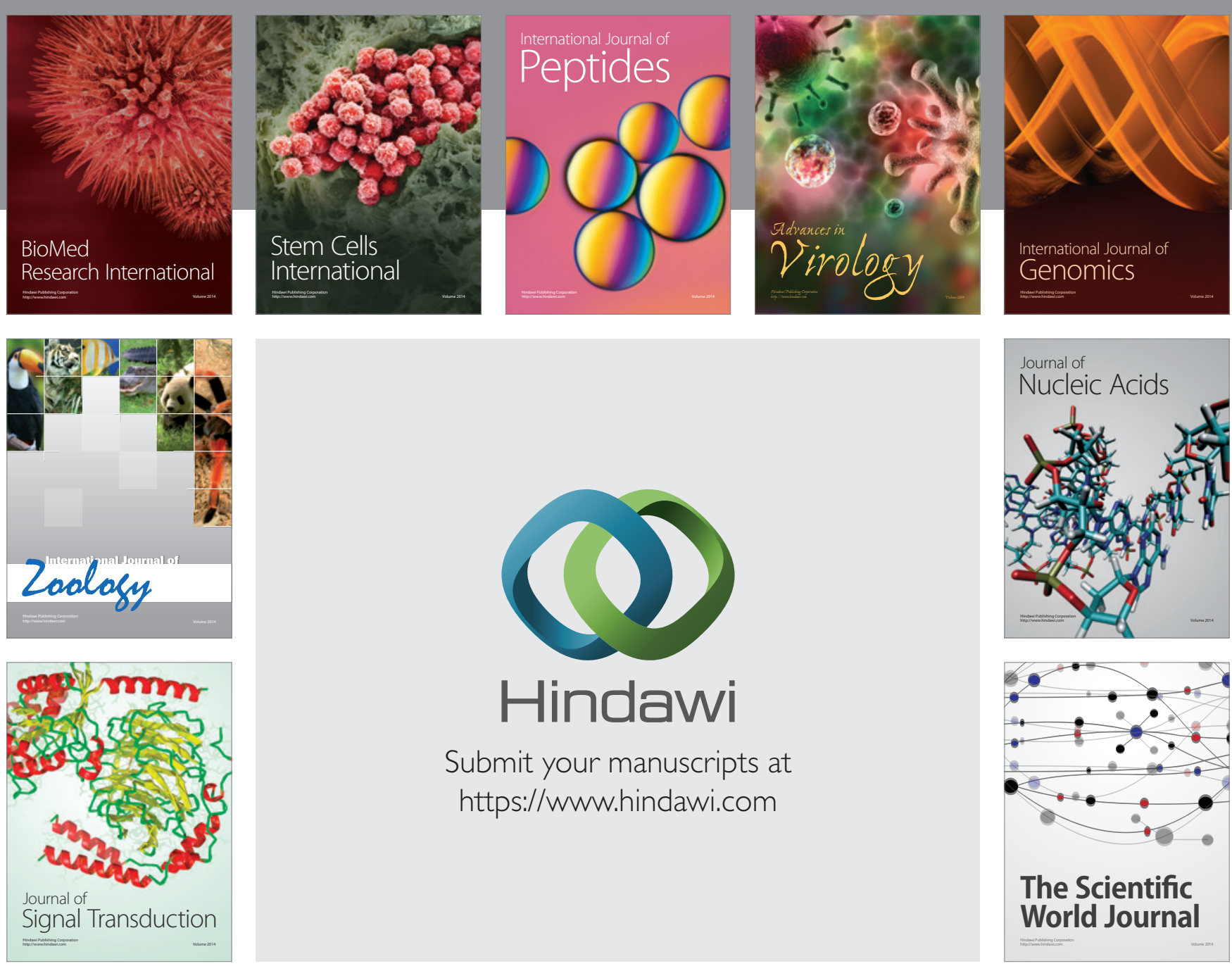

Submit your manuscripts at

https://www.hindawi.com
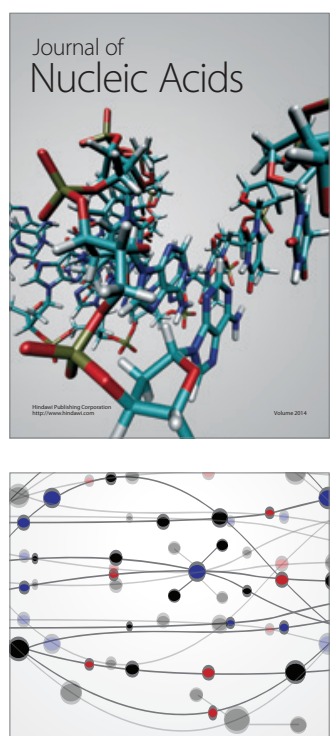

The Scientific World Journal
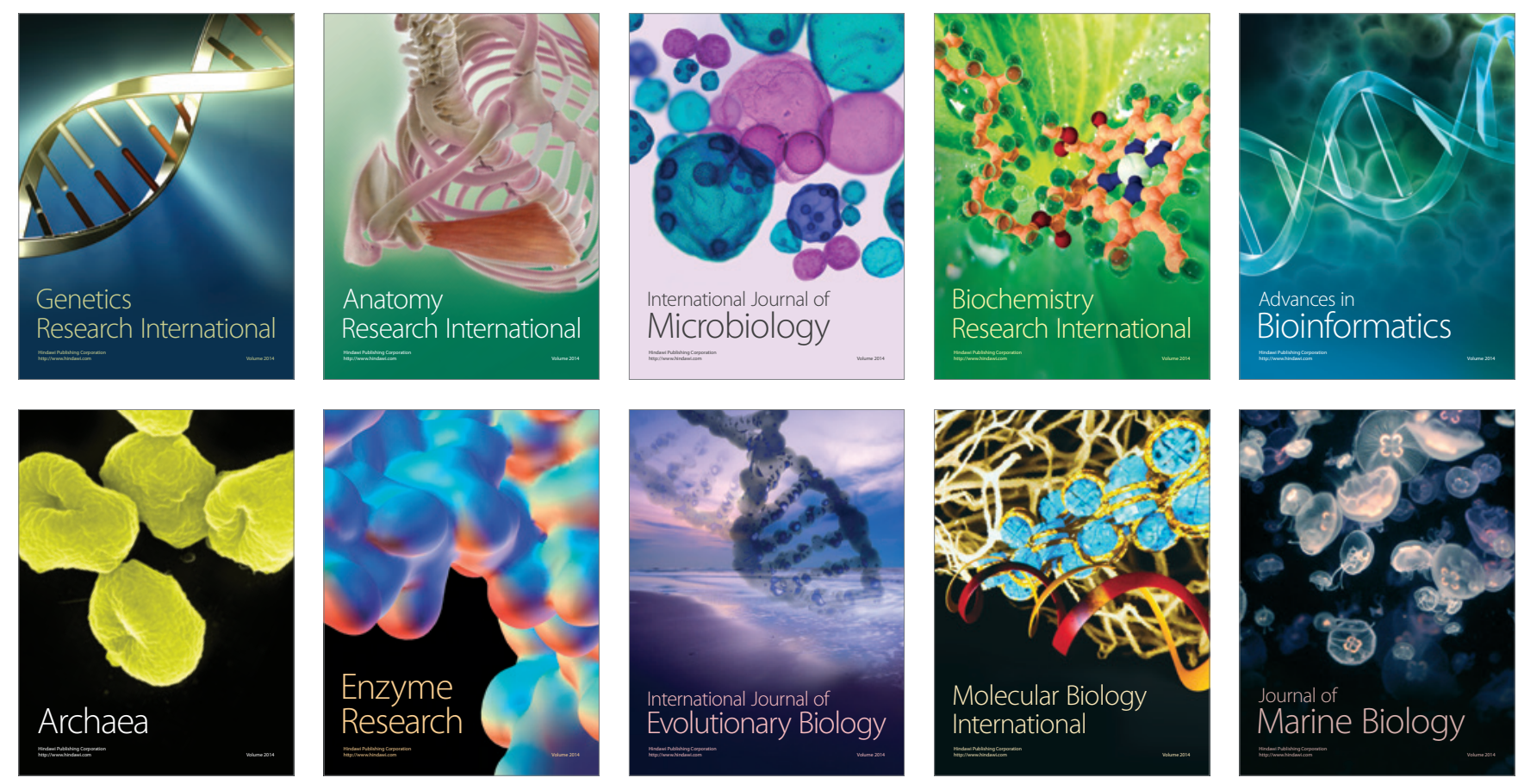